\title{
Steady Patterns of Microparticles Formed by Optical Tweezers
}

\author{
Sheng-Hua Xu ${ }^{1,2}$, Yin-Mei LI ${ }^{1,2^{*}}$, Li-Ren Lou ${ }^{1,2}$, Hong-Tao CHEN ${ }^{1,2}$ and Zhi-Wei SuN ${ }^{3}$ \\ ${ }^{1}$ Open Lab. of Bond Selective Chemistry, University of Science and Technology of China, Hefei, 230026, Anhui, P. R. China \\ ${ }^{2}$ Department of Physics, University of Science and Technology of China, Hefei, 230026, Anhui, P. R. China \\ ${ }^{3}$ National Microgravity Lab, Institute of Mechanics, Chinese Academy of Sciences, Beijing 100080, P. R. China
}

(Received June 18, 2001; accepted for publication September 10, 2001)

In this paper, we propose a method for forming steady patterns of microparticles in a dispersion using optical tweezers. We demonstrate how to control the congregation of particles in a dispersion and to manually fabricate a pattern. The steady pattern may be useful for in-depth research, and the method will have applications in biology and nanotechnology.

[DOI: 10.1143/JJAP.41.166]

KEYWORDS: steady patterns, optical tweezers, microparticles, dispersion, and congregation

Forming steady patterns of $\sim \mu \mathrm{m}$ size particles is of great interest, however an easy way to control and move particles and some conditions to make particles combine firmly are required. Because $\sim \mu \mathrm{m}$ size microparticles are unlike macroparticles that are easy to control, and also unlike atoms that have strong interactions, it is difficult to form steady patterns; no work on this issue has been observed to date.

Before optical tweezers, which utilize radiation pressure of light to control and manipulate microparticles, were invented, it was difficult to manipulate microparticles. Ashkin et al. demonstrated the single-beam gradient-force optical trap for the first time. ${ }^{1,2)}$ A single laser beam enters the microscope and is focused by an objective lens, which has a high numerical aperture (NA). The beam is focused so tightly that the gradient force of the laser beam can manipulate particles in three dimensions. Optical tweezers may now be the most convenient instrument for controlling microparticles during pattern formation.

Many interesting studies have been performed on small particles and optical tweezers. Unsteady patterns of microparticles have been formed by laser-scanning micromanipulation in a dispersion. ${ }^{3)}$ Pattern formation using optical tweezers has been achieved, ${ }^{4)}$ and techniques for rotating microobjects have been improved. ${ }^{5,6)}$ These achievements are both imaginative and interesting. However, the patterns of particles formed will be destroyed if the scanning laser beam is shut off. Steady patterns built by microparticles have never been formed using optical tweezers.

In order to form steady patterns of small particles, there must be chemical conditions such that the particles have strong interactions, as well as instruments for manipulating and moving the particles. Considering the chemical conditions, although the use of laser-scanning optical tweezers may be a good method for manipulating many small particles at one time, it is not suitable for forming steady patterns. It is possible that unnecessary particles will join the patterns, so that the shape of the patterns will be difficult to control. However, the most important point is that, using laser-scanning optical tweezers, it is difficult to form threedimensional instead of planar patterns.

In this letter, we will propose an approach to manipulate microparticles in solution to form steady patterns using a single-beam gradient-force laser trap. Giving the chemical

\footnotetext{
${ }^{*}$ Corresponding author. E-mail: liyinmei@ustc.edu.cn
}

conditions and utilizing the binding force of the particles themselves, we use a single-beam gradient-force optical trap to control and manipulate polystyrene microparticles to form steady spatial patterns. Because the particles in patterns adhere to each other firmly and some particles adhere to the bottom of the sample cell, the patterns are so steady that the particles will not disperse even upon application of adding a $\sim \mathrm{pN}$ force (by optical tweezers); the steady design formed by the optical tweezers remains even when laser source is shut off.

The laser micromanipulation system in this experiment is shown in Fig. 1. The Nd:YVO4 laser beam is a continuous beam of $\mathrm{TEM}_{00}$ mode. The beam was focused on a sample solution through an oil-immersion objective lens $(\mathrm{NA}=$ 1.25 , Olympus). The laser power from the object lens is about $2 \mathrm{mw}$ of which the gradient force of the laser is sufficient to manipulate microscopic particles. Three-dimensional movement is controlled by a computer in the $X Y$ plane, and manually adjusted by an object lens in $Z$ direction. The image is collected by a CCD camera and displayed by a computer. The dispersion used in our experiment is the product of Duke Scientific Corporation, USA. The polystyrene balls in dispersion are about $2 \mu \mathrm{m}$ in diameter $(2.013 \pm 0.025 \mu \mathrm{m})$. The system is very simple, but the key to our technique in this experiment is the choice of the concentration of the electrolyte in dispersion.

The electrolyte, more precisely the ion in solution, may

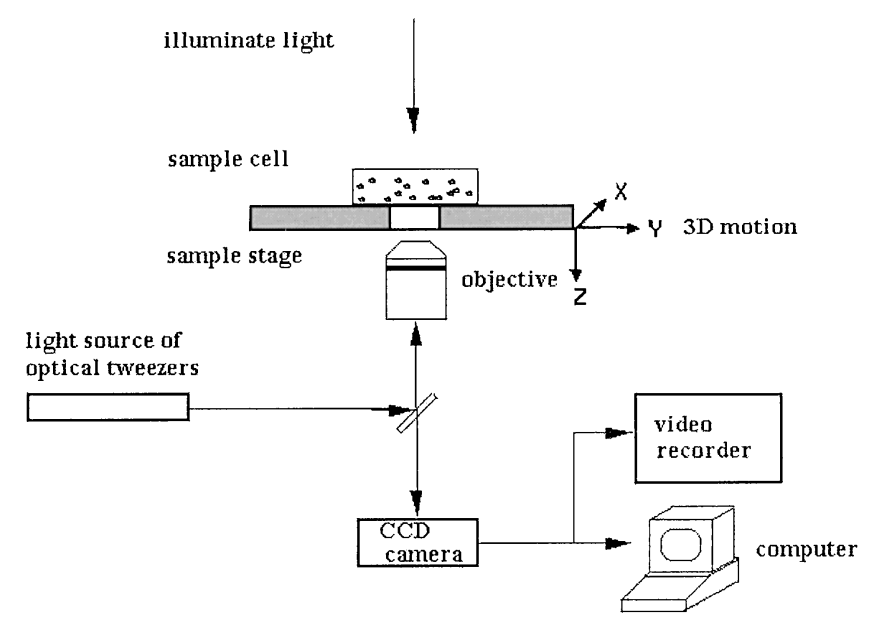

Fig. 1. A schematic diagram of the instrument used in experiments. 
change the charge distribution on the surface of small particles in the dispersion, and then inflect the interaction of particles. The dispersion may be stable so that the particles in it do not congregate if there is no electrolyte. The polystyrene solution also has this characteristic. In the polystyrene solution used by us, when the concentration of the electrolyte is very high, particles in dispersion will congregate and deposit on the sample surface rapidly. ${ }^{7)}$ On the other hand, if the concentration is too low, the coupling of the particles would be pure repulsion. Then we cannot even use optical tweezers to make particles congregate; neither will it be possible to form a steady pattern. Thus, in our experiment, we take a concentration that is neither too high nor too low, so that particles cannot gather quickly by their own Brownian motion, but if we use optical tweezers to bring them into contact, they will adhere to each other easily.

We use different concentrations of $\mathrm{NaCl}$ in polystyrene solution in the experiment and find that $0.5 \mathrm{~mol} / \mathrm{l} \mathrm{NaCl}$ is very appropriate for our experiment. Under this concentration, the particles are mostly single and do not gather quickly, which makes pattern formation easy. We utilize an optical trap to control and manipulate single polystyrene particles so that they adhere to the bottom of sample cells or other particles. After the controlled particles adhere to the bottom of cells or other particles, we remove the optical tweezers and trap another particle to repeat the process. With this method, steady patterns of particles are formed.

Figure 2 is a pattern of "LOT" (laser optical tweezers) formed by $\sim 2 \mu \mathrm{m}$ polystyrene particles on the bottom of the sample cell. Particles in the pattern adhere to the bottom of the cell and are on the same horizontal plane. The formed pattern is steady due mostly to the interaction between particles and the surface of the bottom.

Figure 3 demonstrates spatial patterns of $\sim 2 \mu \mathrm{m}$ polystyrene latex particles. Figure 3(a) is the spatial pattern of " $\mathrm{X}$ " formed by 16 polystyrene particles. Under the pattern, there are four other particles, which form a vertical column and make the pattern " $\mathrm{X}$ " $8 \mu \mathrm{m}$ up from the bottom. Figure 3 (b), in which the line represents the sample cell bottom surface, is the schematic front view of this pattern. We can see from Fig. 3(a) that the particles of " $X$ " are of the same shape, and can conclude that they are in the same imaging plane, that is, the particles are in the plane parallel to the sample cell bottom. As mentioned above, the pattern is some distance from the bottom due to the four particles under the pattern. If we put a different number of particles under the

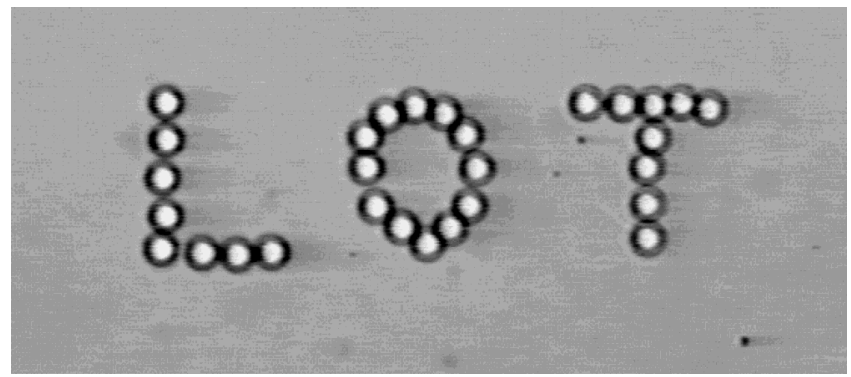

Fig. 2. Pattern formation "LOT" of particles on the bottom of the sample cell. It is steady due to the interaction between particles and sample cells. (a)

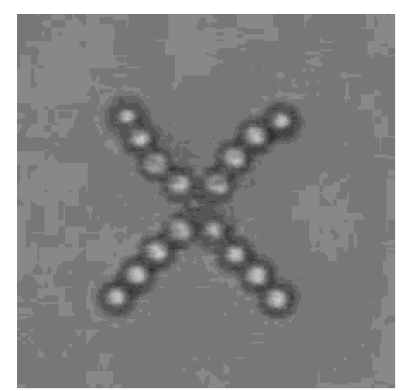

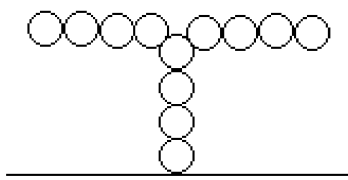

(b)
Fig. 3. Pattern " $\mathrm{X}$ " formed by polystyrene particles. (a) Top view of pattern " $\mathrm{X}$ " formed by 16 polystyrene particles $2 \mu \mathrm{m}$ in size.The interactions between particles themselves make the pattern steady. (b) Schematic front view of " $X$ ". The line represents the sample cell surface. The four particles in the vertical direction form the column under " $\mathrm{X}$ ".

pattern, we can form any pattern at any distance from the bottom. This formed pattern is steady due mostly to the interaction of particles themselves, which is not the case in Fig. 2.

We can also form a pattern not parallel to the bottom. Figure 4 shows a formed pattern of " $A$ " of 24 particles. Because the particles are not the same distance from the bottom, they look very different from each other although they are all the same size $(\sim 2 \mu \mathrm{m})$. In fact, only the apex of "A" adheres to the bottom. The other particles are combined with adjacent particles. Further away from the acme, the particles are more distant from the bottom. Other forms of steady patterns can also be produced utilizing this method.

In this study, it is clearly shown that optical tweezers are extremely useful since any steady spatial pattern of particles can be produced. Without optical tweezers, particles in a dispersion will congregate in a random way, but utilizing this technique, the congregation becomes controllable and the fabrication of an artificial pattern of particles is possible. Moreover, particles other than polystyrene and electrolytes other than $\mathrm{NaCl}$ can also be used, so that the experiment may have applications in many fields.

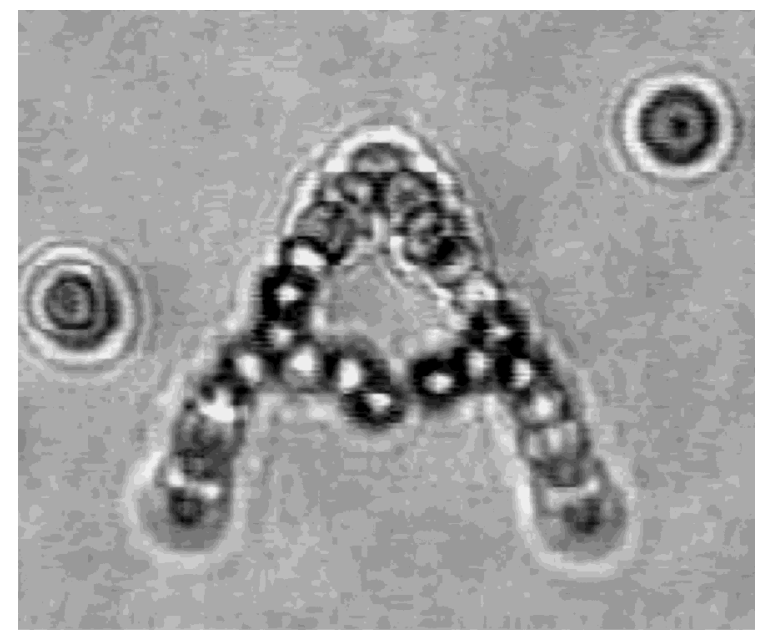

Fig. 4. Pattern "A" formed by 24 particles. Only the apex of "A" adheres to the bottom. 
It should be emphasized here that the patterns fabricated are steady. We expect that our method of manufacturing steady patterns will play a major role in advanced research into the chemical, physical, mechanical and electrical characteristics of fine particles and their artificial clusters. If the particles are all biological cells, our method will be unique in researching the interaction and communication between cells, which is important in biology. The method is also useful for $\sim \mu \mathrm{m}$ and $\sim$ nm assemblage. In $\mathrm{nm}$ science, optical tweezers will become a well-used instrument for achieving this assemblage. Further studies on their application will be useful in the near future.

This study was supported by National Natural Science
Foundation of China (No. 19890380, No.10072062).

1) A. Ashkin: Phys. Rev. Lett. 24 (1970) 156.

2) A. Ashkin, J. M. Dziedzic, J. E. Bjorkholm and S. Chu: Opt. Lett. 11 (1986) 288.

3) K. Sasaki, M. Koshioka, H. Misawa, N. Kitamura and H. Masuhara: Jpn. J. Appl. Phys. 30 (1991) 907.

4) P. C. Mogensen and J. Glückstad: Opt. Commun. 175 (2000) 75.

5) E. Higurashi, H. Ukita, H. Tanaka and O. Ohguchi: Appl. Phys. Lett. 64 (1994) 2209.

6) Z. P. Luo, Y. L. Sun and K. N. An: Appl. Phys. Lett. 76 (2000) 1779.

7) P. C. Hiemenz: Principles of Colloid and Surface Chemistry (Marcel Dekker, New York, 1977). 